\title{
Generating multiple-pulse bursts for enhanced fluorescence detection
}

Shumilov, Dimytro; Rich, Ryan M.; Gryczynski, Ignacy; Raut, Sangram; Gryczynski, Karol; Kimball, Joe; Doan, Hung ; Sørensen, Thomas Just; Laursen, Bo Wegge; Borejdo, Julian; Gryczynski, Zygmunt

\section{Published in:}

Methods and Applications in Fluorescence

DOI:

$10.1088 / 2050-6120 / 2 / 2 / 024009$

Publication date:

2014

Document version

Publisher's PDF, also known as Version of record

Citation for published version (APA):

Shumilov, D., Rich, R. M., Gryczynski, I., Raut, S., Gryczynski, K., Kimball, J., Doan, H., Sørensen, T. J., Laursen, B. W., Borejdo, J., \& Gryczynski, Z. (2014). Generating multiple-pulse bursts for enhanced fluorescence detection. Methods and Applications in Fluorescence, 2, [024009]. https://doi.org/10.1088/2050$6120 / 2 / 2 / 024009$ 
Generating multiple-pulse bursts for enhanced fluorescence detection

This content has been downloaded from IOPscience. Please scroll down to see the full text. 2014 Methods Appl. Fluoresc. 2024009

(http://iopscience.iop.org/2050-6120/2/2/024009)

View the table of contents for this issue, or go to the journal homepage for more

Download details:

IP Address: 130.225.98.216

This content was downloaded on 13/03/2015 at 10:50

Please note that terms and conditions apply. 


\title{
Generating multiple-pulse bursts for enhanced fluorescence detection
}

\author{
Dmytro Shumilov ${ }^{1}$, Ryan M Rich ${ }^{2}$, Ignacy Gryczynski ${ }^{2}$, Sangram Raut ${ }^{1,2}$, \\ Karol Gryczynski ${ }^{2}$, Joe Kimball ${ }^{1}$, Hung Doan ${ }^{1}$, Thomas J Sørensen ${ }^{3}$, \\ Bo W Laursen ${ }^{3}$, Julian Borejdo ${ }^{2}$ and Zygmunt Gryczynski ${ }^{1,2}$ \\ 1 Department of Physics \& Astronomy, Texas Christian University, Fort Worth, TX 76129, USA \\ 2 Department of Molecular Biology and Immunology, Center for Commercialization of Fluorescence \\ Technologies, University of North Texas Health Science Center, Fort Worth, TX 76107, USA \\ ${ }^{3}$ Nano-Science Center \& Department of Chemistry, University of Copenhagen, Universitetsparken 5, \\ DK-2100 København Ø, Denmark
}

E-mail: z.gryczynski@tcu.edu

Received 24 January 2014, revised 25 March 2014

Accepted for publication 7 April 2014

Published 14 May 2014

\begin{abstract}
The signal-to-background ratio is the limiting factor for fluorescence based detection, sensing, and imaging. A typical background signal will include direct scattering of excitation and Raman scattering of the sample as well as autofluorescence from the sample and additives. To improve the signal-to-background ratio, fluorophores of high brightness and/or high concentration of the fluorophores need to be used. Most of the background is instantaneous and short-lived (picosecond to nanosecond time scale), and using long-lived fluorescence probes combined with time-gated detection allows for significant suppression of unwanted background. Unfortunately, this approach requires substantial sacrifice of the probe signal in order to sufficiently filter the background unless the fluorescence lifetime of the probe is very long. However, long lived probes like ruthenium bipyridyl have relatively low brightness compared to probes that have shorter, 10-30 ns fluorescence lifetimes.

We recently presented an approach based on bursts of multiple pulses that allowed for high probe signal amplification using long-lived ruthenium based probe $(\mathrm{Ru})$ and an $80 \mathrm{MHz}$ repetition-rate laser excitation. Unfortunately, Ru represents an extreme case for probe lifetime, and a probe with a shorter lifetime of $20 \mathrm{~ns}$ will require excitation from a pulsed source with much higher repetition rate to significantly enhance its signal. Such high repetition rates are not possible to generate with most of today's available electronics. In this report we present new approaches to optimize and generate bursts of pulses with high repetition rate within the burst and no need for new or improved electronics. The high repetition rates originate from a low-repetition source and are highly tunable. We demonstrate that a burst of 2-10 pulses spaced $3 \mathrm{~ns}$ apart (corresponding to a 'burst repetition rate' of $330 \mathrm{MHz}$ ) allows for high signal enhancement of the $20 \mathrm{~ns}$ probe over the sub-nanosecond/nanosecond background. Such an approach can be applied for any sensing format, allowing much higher sensitivity for detection. Since the energy of a single pulse is spread over a few pulses in the burst, the fluorophore's photostability also improves.
\end{abstract}

Keywords: fluorescence detection, multi-pulse, long lived fluorophores, fluorescence based imaging, fluorescence microscopy

(Some figures may appear in colour only in the online journal) 

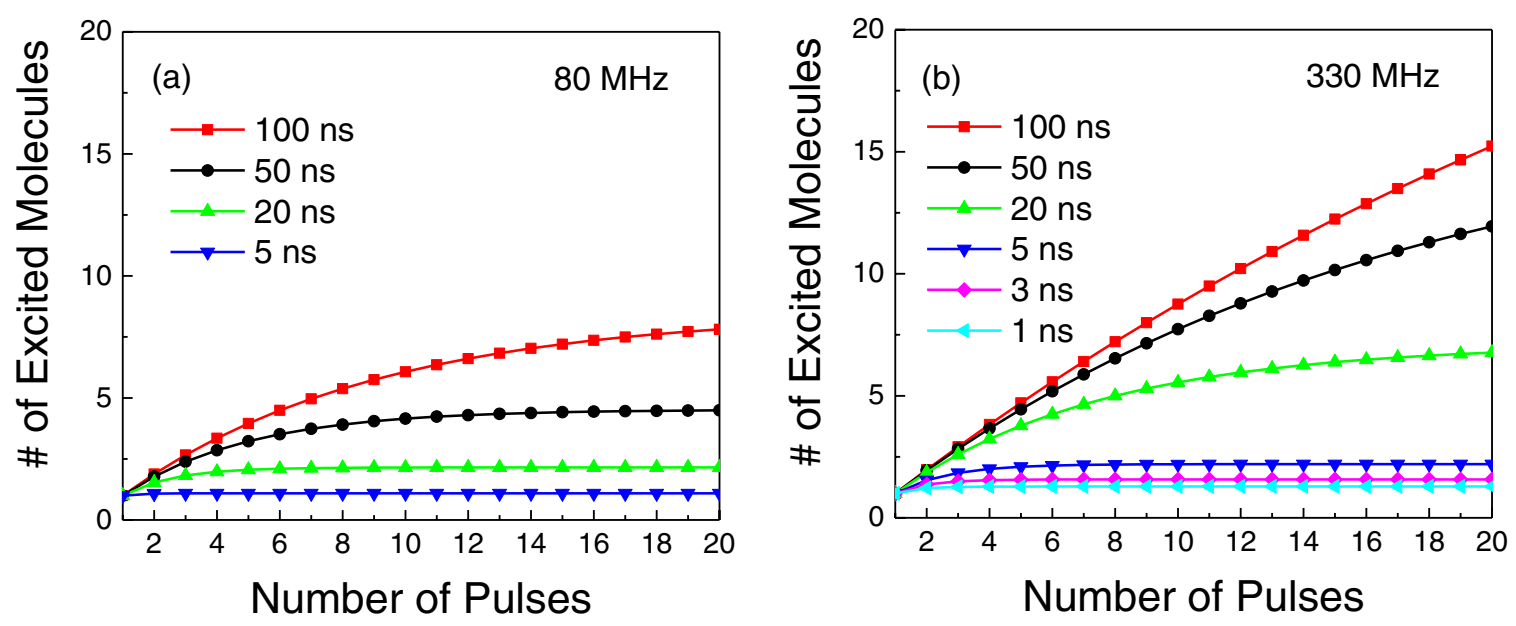

Figure 1. Number of molecules in the excited state as a function of number of pulses for $80 \mathrm{MHz}$ (left panel) and $330 \mathrm{MHz}$ (right panel) RRs, respectively.

\section{Introduction}

Fluorescence is considered as one of the most sensitive detection technologies, quickly entering various fields from environmental sensing to biomedical diagnostics [1-3]. With the sensitivity level reaching nanomolar and picomolar concentrations, the fundamental problem for fluorescence based detection becomes the background signal that, in many occasions, can be comparable to or overwhelm the signal from the probe. To distinguish a desirable probe signal from the background, one would typically need to increase probe concentration, which is not always possible or physiologically acceptable. Since autofluorescence in biologically relevant samples quickly decreases with the wavelength, a big effort in the field has been directed toward developing near infra-red (NIR) probes [4-7]. Additional improvement in the signal-to-noise ratio can be achieved by using long-lived probes and time-gated detection [8-10]. The fluorescence signal of probes with lifetimes significantly longer than the fluorescence lifetime of the background decays much slower than the background perturbation. Therefore opening the detector a short time after the excitation pulse greatly attenuates the background contribution without significant loss in the probe signal. This approach has shown an excellent improvement in the signal-to-noise ratio for many long-lived emitters. Unfortunately most of the probes in the visible/ red spectral range that have long fluorescence lifetime also have relatively low extinction coefficients and typically very low quantum yields. Shifting the probe absorption even farther toward the NIR spectral range allows for a significant increase in the extinction coefficient and quantum yield, but at the same time, the fluorescence lifetime is shortened, thus making it harder to employ time-gated detection to remove the background. In effect, efforts to increase the extinction coefficient and/or quantum yield gave only limited results leaving us without any possibilities for increasing the brightness of the probe.

We recently reported a method capable of selectively enhancing the signal-to-noise ratio when using long-lived probes [11]. Using a long-lived ruthenium bipyridyl $(\mathrm{Ru})$ probe and multiple-pulse excitation (burst of pulses) we were able to achieve significant signal-to-noise enhancement in tissue imaging experiments. Since the fluorescence lifetime of $\mathrm{Ru}$ is over $350 \mathrm{~ns}$, a pulse burst with an internal repetition rate (RR) of $80 \mathrm{MHz}$ was sufficient to selectively enhance $\mathrm{Ru}$ excitation more than an order of magnitude over the background. The long lifetime of $\mathrm{Ru}$ is also excellent for the time-gated detection approach, which can be simultaneously applied with multi-pulse excitation, but at the same time, this very long fluorescence lifetime significantly slows the scanning rate for fluorescence lifetime-imaging microscopy (FLIM) experiments. To increase the speed for image collection it would be desirable to apply bursts of pulses to fluorescence probes with much shorter fluorescence lifetimes on the order of 10-50 ns. Since the typical fluorescence lifetime of the background fluorescence (cells and intrinsic tissue fluorescence) is $1-5 \mathrm{~ns}$ [11-13], a signal from a probe with fluorescence lifetime longer than 10 ns should be significantly elevated above the background when excited with bursts of pulses that have a high internal RR. Unfortunately most commercial laser systems work with an $80-90 \mathrm{MHz}$ maximum rep rate, resulting in a time gap between pulses of 11-12.5 ns. For a fluorophore that has a fluorescence lifetime of $\sim 20 \mathrm{~ns}$, the possible enhancement with an $80 \mathrm{MHz}$ rep rate in the burst will be relatively low. Various strategies/technologies for lowering the laser RR are well established, but increasing the RR to $200-500 \mathrm{MHz}$ is much more problematic.

In this report we present simple methods to generate bursts of pulses with a desirable high and tunable RR within the burst from a train of low repetition single pulses (like pulses from a typical laser diode generated with RR $20 \mathrm{MHz}$ or lower). We discuss a theoretical approach to optimize the spacing between pulses in the burst (apparent internal RR) as a function of the probe and background lifetimes. Depending on the fluorescence lifetime of the probe, we can tune the temporal spacing between pulses within the burst (RR within the burst) to achieve the maximum ratio between probe and background signal. We present a simple example in which the signal of the AzaDiOxaTriAnulenium (ADOTA) [12-14] probe with a fluorescence lifetime of $20 \mathrm{~ns}$ is increased more than $300 \%$ 


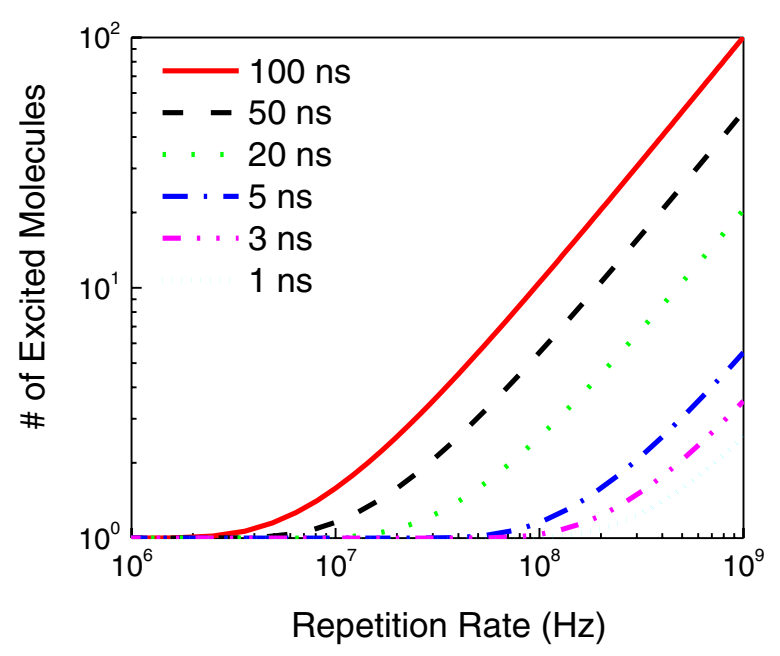

Figure 2. Number of molecules in the excited state after infinitely long bursts as function of RR in the burst for various fluorescence lifetimes.

over the short-lived background by just generating 3-5 pulse bursts in place of a single pulse experiment.

Such an approach opens new possibilities for enhanced detection, sensing, and imaging with the use of only moderately long-lived fluorophores. Probes with fluorescence lifetimes in the order of 10-40 ns will typically have much higher brightness than long-lived ruthenium based probes and a few fold increase of the signal yields very sensitive detection. Significant increase in apparent brightness of the probe over the background opens new possibilities for chemical and biomedical sensing, biomedical diagnostics, and cellular and tissue imaging applications.

\section{Theoretical model}

Excluding single molecule approaches, the majority of fluorescence and fluorescence based sensing/imaging experiments are conducted under the condition for which only a very small fraction of the fluorescent molecules can be excited with a single pulse. In commonly used cuvettes or solid samples, even a well collimated laser beam will contain millions of dye molecules in the excitation volume where only a small fraction can be excited by a single excitation pulse. Even a typical confocal volume used for cellular and tissue imaging contains a significant number of fluorophores and only a small portion of them are excited with a single pulse. In other words, the conditions for a typical fluorescence experiment are very different from experiments like single molecule or fluorescence correlation spectroscopy (FCS), where the main effort is to limit the number of observed molecules by drastically limiting the probe concentration and excitation volume.

Consider a short, single pulse that excites $N_{e}$ molecules in the sample out of the total number of fluorophores available in the excitation volume of $N_{\mathrm{T}}$ where $N_{\mathrm{T}}>>N_{\mathrm{e}}$. The number of excited molecules decays quickly depending on the molecules' fluorescence lifetime, $\tau$, as $N_{\mathrm{e}}(t)=N_{\mathrm{e}} \mathrm{e}^{-t / \tau}$. The number of emitted photons at any moment in time is proportional to

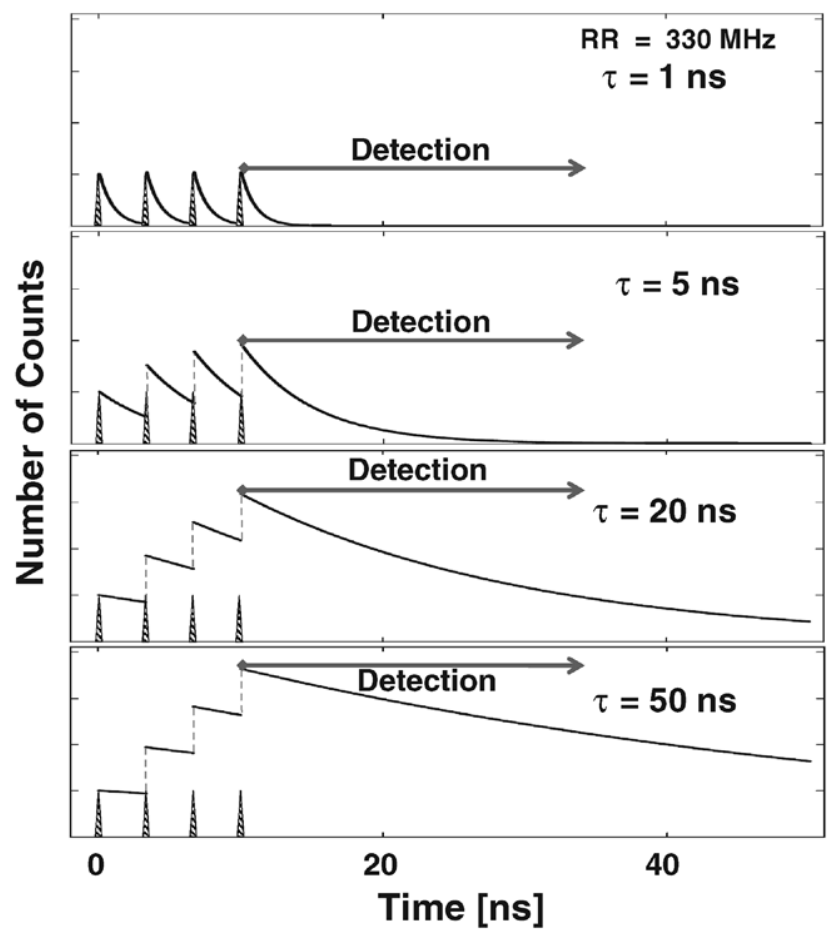

Figure 3. Number of molecules in the excited state expected with four pulse burst spaced by $3 \mathrm{~ns}(330 \mathrm{MHz} \mathrm{RR})$ for four fluorescence lifetimes of 1, 5, 20 and $50 \mathrm{~ns}$.

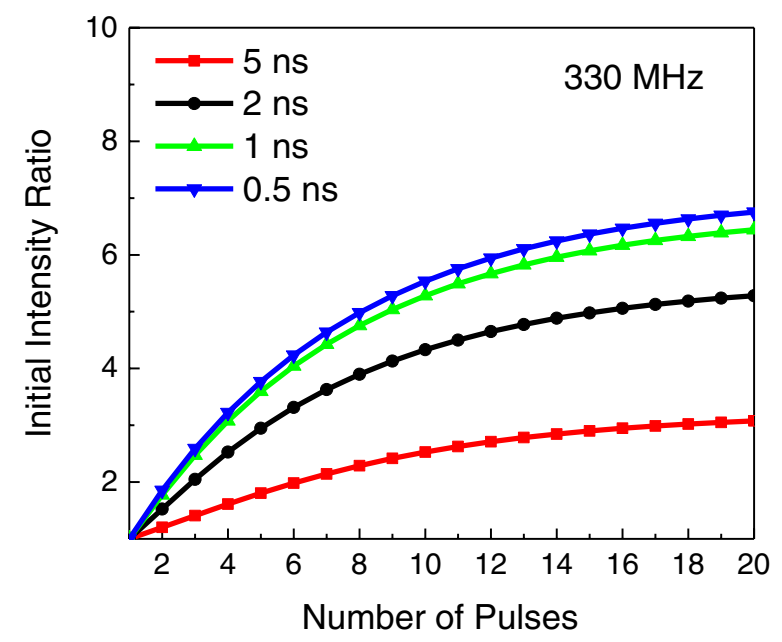

Figure 4. Ratio of initial intensities for fluorescence lifetime of $20 \mathrm{~ns}$ versus lifetimes of 5,2,1 and $0.5 \mathrm{~ns}$.

the total number of molecules in the excited state at a given time. Consequently the observed instantaneous fluorescence intensity, $I(t)$, is proportional to the number of molecules in the excited state $\left(I(t) \sim N_{\mathrm{e}}(t)\right)$. For low RRs, when the population of excited molecules decays completely before the arrival of the next pulse, the average steady-state intensity will be proportional to the pulse intensity and RR of the laser, or in fact, the number of pulses per unit time. Most fluorophores decay within a few nanoseconds and typical RR that are $80 \mathrm{MHz}$ or lower give a pulse separation $\Delta t>12.5 \mathrm{~ns}$. Consequently molecules that have a fluorescence lifetime of less than $4 \mathrm{~ns}$ excited by one pulse decays almost completely 

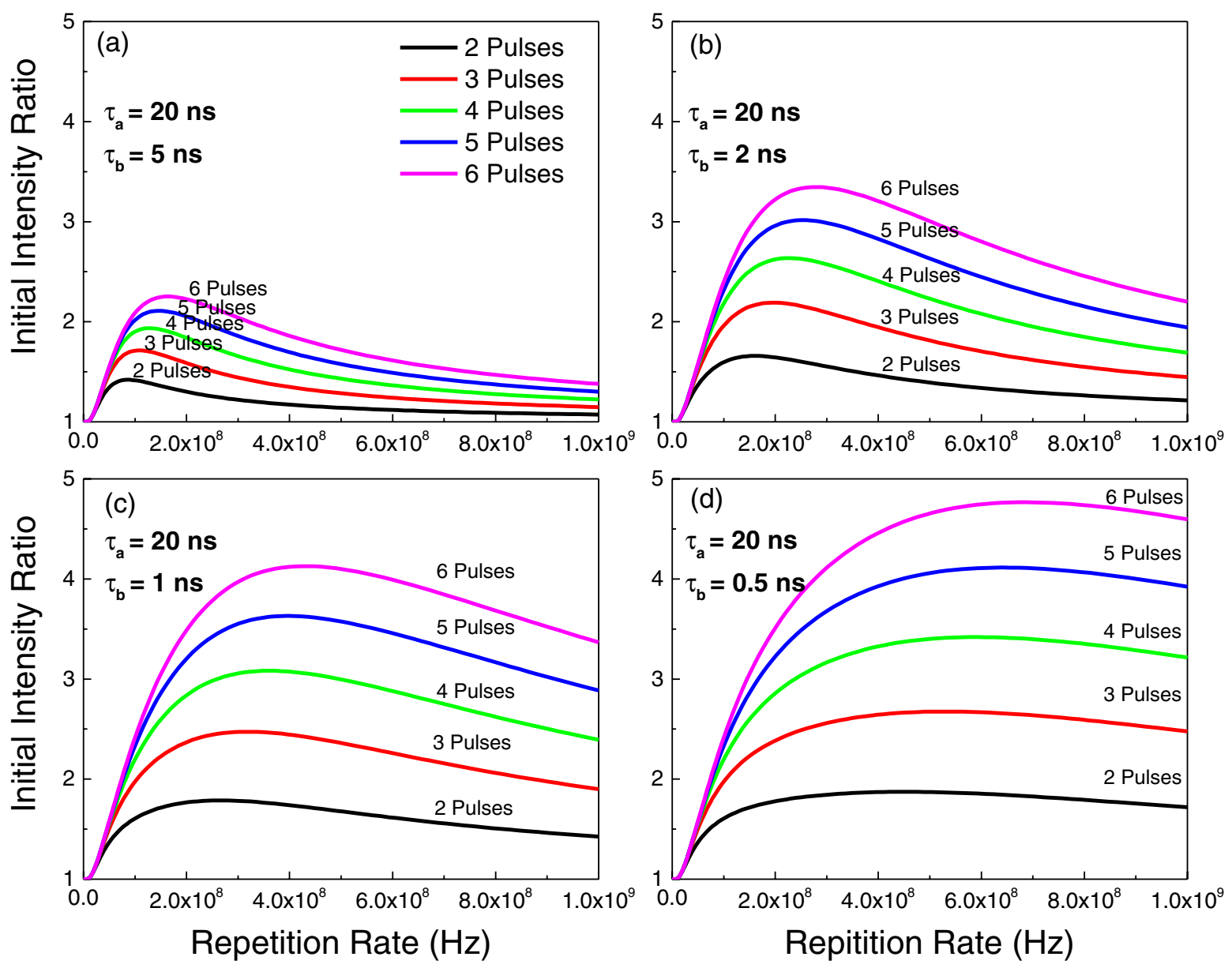

Figure 5. Relative signal enhancement for $20 \mathrm{~ns}$ sample lifetimes and background lifetimes of $5 \mathrm{~ns}(a), 2 \mathrm{~ns}(b), 1 \mathrm{~ns}(c)$ and $0.5 \mathrm{~ns}(d)$, respectively.

before the next pulse arrives. When the fluorescence lifetime is longer than the pulse separation $(\tau>\Delta t)$, the excited state population decays only partially and new molecules excited by the next incoming pulse are added to the molecules still in the excited state. In such a system, the number of molecules in the excited state will increase with each pulse reaching the equilibrium when the number of molecules excited by a single pulse is equal to the number of molecules that return to the ground state over the time interval equal to pulse separation, $\Delta t$. One can calculate the number of molecules in the excited state by analyzing subsequent excitations. The first pulse excites $N_{\mathrm{e}}$ molecules that, for simplicity, is much lower than the total number of fluorophores in the excitation volume $N_{\mathrm{e}}<<N_{\mathrm{T}}$. When the second pulse arrives, the number of molecules remaining in the excited state is $N_{1}=N_{\mathrm{e}} \mathrm{e}^{-1 / \tau \cdot \mathrm{RR}}$ and the total number of excited molecules immediately after second pulse is: $N_{\mathrm{e}}^{1}=N_{1}+N_{\mathrm{e}}$ assuming that the second pulse excites the same number of molecules. In fact, the number of fluorophores available in the excitation volume is lower $\left(N_{\mathrm{T}}-N_{1}\right)$, but for our consideration we assume $N_{\mathrm{T}}-N_{1} \approx N_{\mathrm{T}}$. Extending this for $n$ pulses, we have a geometrical series, and the number of molecules in the exited state after $n$ pulses will be:

$$
N_{\mathrm{eT}}^{n}=N_{\mathrm{e}} \frac{1-\mathrm{e}^{-n / \tau \cdot \mathrm{RR}}}{1-\mathrm{e}^{-1 / \tau \cdot \mathrm{RR}}}
$$

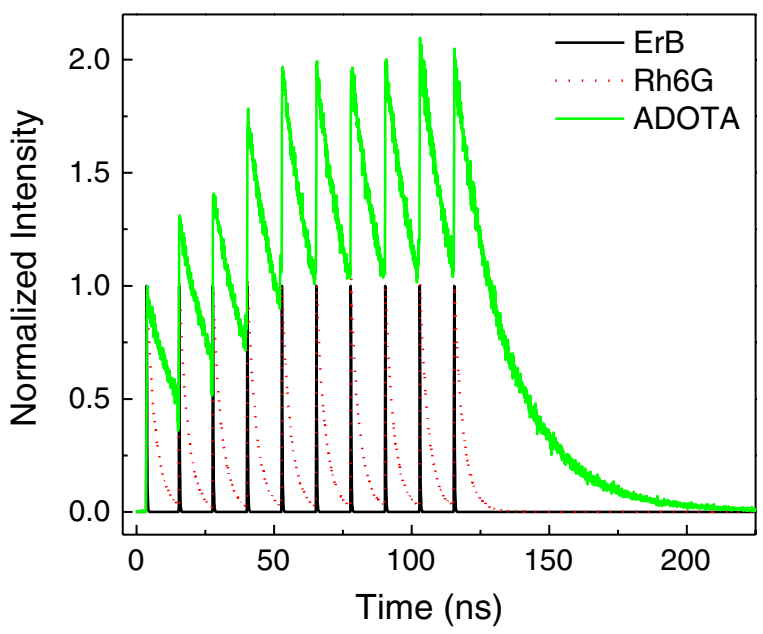

Figure 6. Signal measured with 10 pulses burst of $80 \mathrm{MHz}$ RR.

And consequently for an infinite number of pulses we have the semi-equilibrium condition where:

$$
N_{\mathrm{eT}}=\frac{N_{\mathrm{e}}}{1-\mathrm{e}^{-1 / \tau \cdot \mathrm{RR}}}
$$

Figure 1 shows the number of molecules in the excited state as a function of the number of pulses with RR of 80 and $330 \mathrm{MHz}$. For an $80 \mathrm{MHz}$ RR and a moderately long fluorescence 


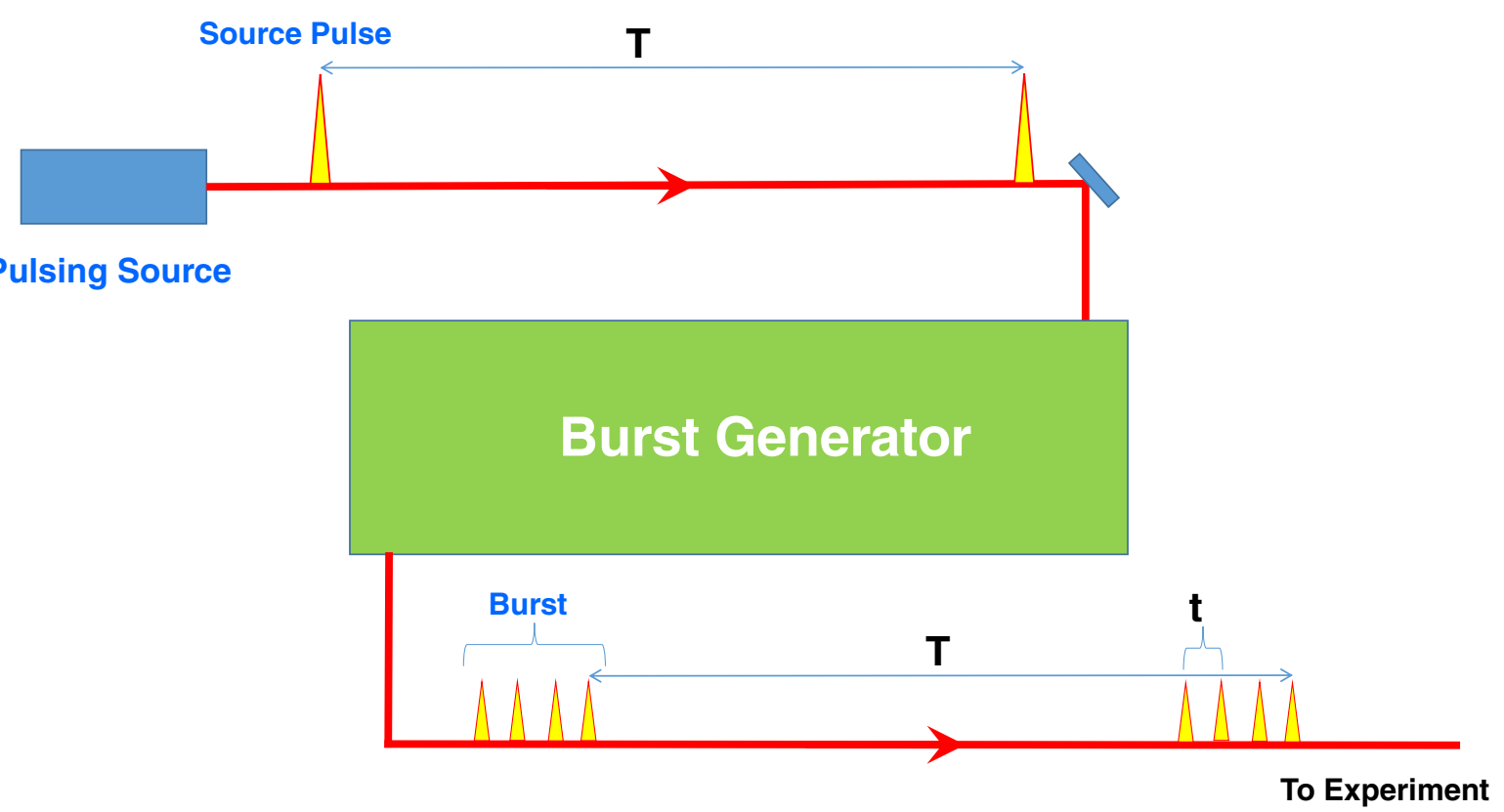

Figure 7. Concept for burst generator from a single pulse.

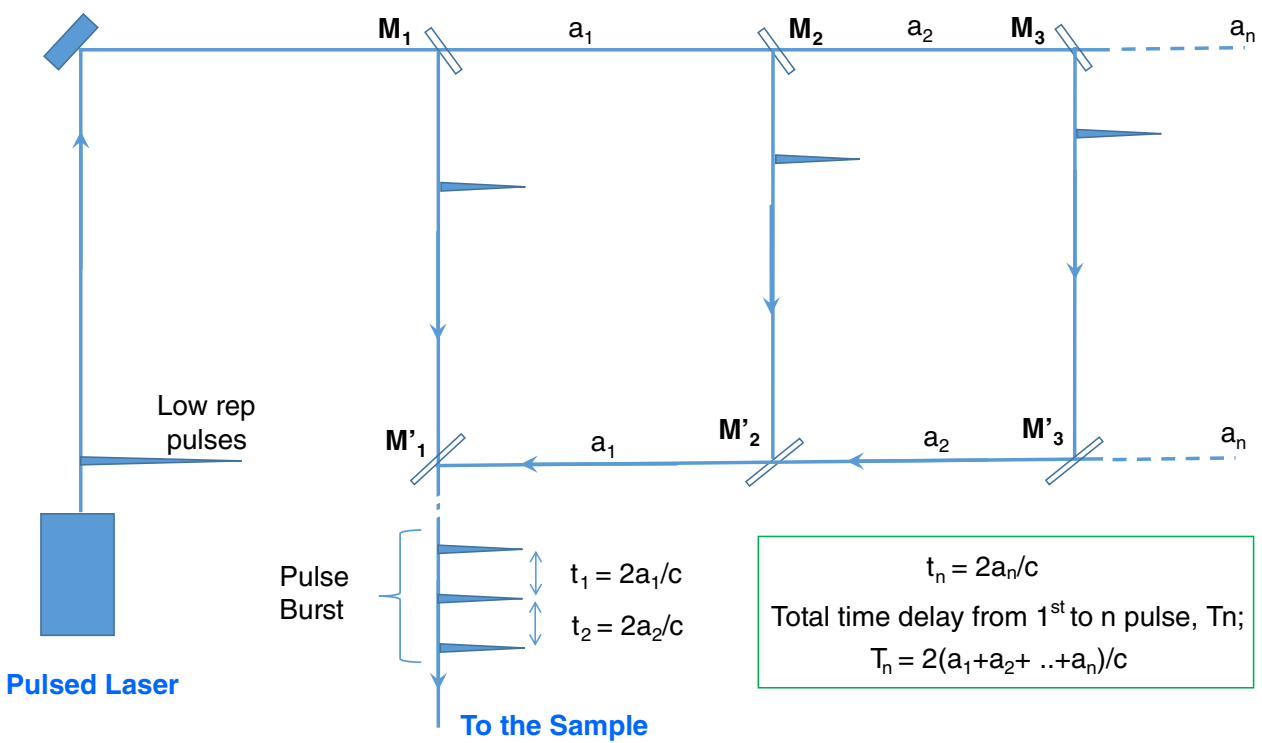

Figure 8. Schematic for a single pulse splitting and delaying. The delay is calculated as distance $\left(a_{1}+\ldots\right)$ divided by speed of light, $c$.

lifetime of $20 \mathrm{~ns}$ or shorter, the increase in molecules in the excited state is small. However for a $330 \mathrm{MHz} R \mathrm{R}$ the number of molecules in the excited state quickly increases as a function of the number of pulses for lifetimes of $20 \mathrm{~ns}$ and longer. But the effect is negligible when the fluorescence lifetime is $5 \mathrm{~ns}$ or shorter. In figure 2 , we present the maximum number of molecules in the excited state as a function of rep rate in the burst for various fluorescence lifetimes. For low RR when pulse separation, $\Delta t$, is $\Delta t=1 / \mathrm{RR}>>\tau$ the number of molecules in the excited state after each pulse does not depend on the RR and is the same for each fluorescence lifetime. Excited fluorophores decay to the ground state well before the arrival of each consecutive pulse. As the RR approaches the $1 / \tau$ value, not all fluorophores are able to decay and each consecutive pulse adds to the un-decayed population of the excited state.
After a large number of pulses the system reaches semi-equilibrium where the number of molecules in the excited state after each pulse is equal to $N_{\mathrm{eT}}$ given by equation (1). Between two consecutive pulses, a number of $N_{\mathrm{e}}$ molecules will decay to the ground state. Further increase of the RR increases the population of the excited state, and for significantly higher $\mathrm{RR}$, the excited state population is an exponential function of RR (linear dependence in a logarithmic scale in figure 2). After a given number of pulses, the number of molecules in the excited state will depend on the ratio of fluorescence lifetime, $\tau$, to $1 / \mathrm{RR}$.

In a typical experiment we will not use a large (infinite) number of pulses in the burst, and it is interesting to consider how the number of excited molecules changes for limited number of pulses in the burst. Figure 3 shows the number 


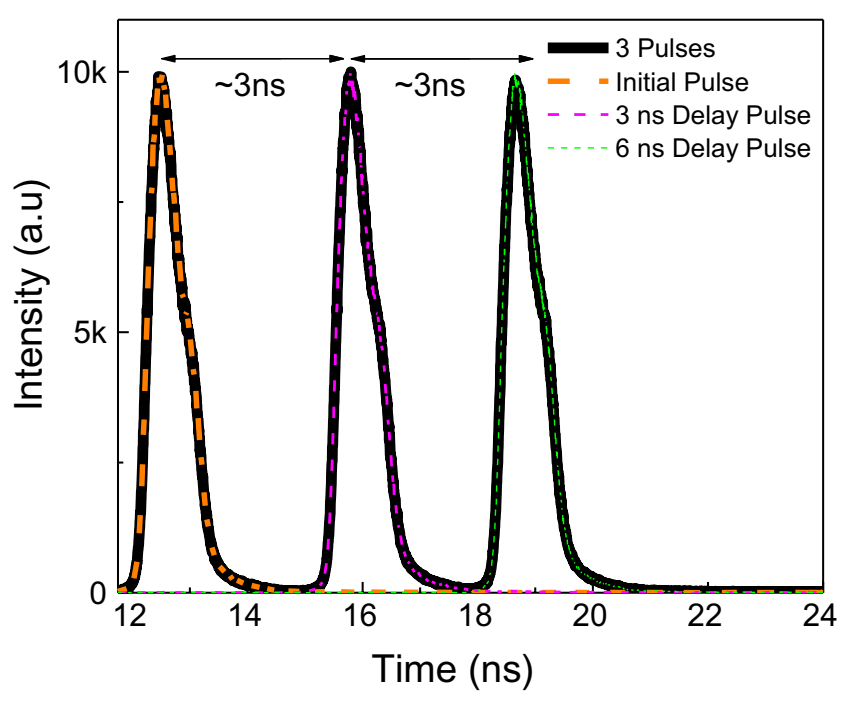

Figure 9. Traces of three pulses generated with the line shown in figure 7 and measured with photo-multiplier tube (PMT). Each color represents individual pulse and black color overlays three pulses measured simultaneously.

of excited molecules with a four pulse burst when consecutive pulses are separated by $3 \mathrm{~ns}(330 \mathrm{MHz} \mathrm{RR})$ for a series of fluorescence lifetimes of 1, 5, 20 and 50ns. It is clear that for lifetimes of $20 \mathrm{~ns}$ and longer the initial intensity is highly increased (over threefold) while for lifetimes shorter than $5 \mathrm{~ns}$ the increase is negligible. For a pulse separation of $12.5 \mathrm{~ns}$ ( $80 \mathrm{MHz} \mathrm{RR})$ the increase becomes significant only when probe lifetimes are longer than $50 \mathrm{~ns}$ (data not shown) [11].

Figure 4 shows a simulation for initial signal ratios between the long lived $(20 \mathrm{~ns})$ fluorophores and varied, short lived background contributions with lifetimes of $0.5 \mathrm{~ns}, 1 \mathrm{~ns}, 2 \mathrm{~ns}$, and $5 \mathrm{~ns}$, respectively as a function of the number of pulses in the burst with an internal RR of $330 \mathrm{MHz}$. It is clear that for the probe with fluorescence lifetime of $20 \mathrm{~ns}$, the initial intensity highly increases with increasing number of pulses (over threefold already for four pulses) when lifetimes of the background are shorter than $2 \mathrm{~ns}$. The $20 \mathrm{~ns}$ fluorescence lifetime is accessible with our ADOTA probes [13, 14] and the major components of typical backgrounds range from 0.3 to $2.0 \mathrm{~ns}$ [12]. For fluorescence lifetimes of the background shorter than $2 \mathrm{~ns}$, the enhancement is practically linear for the first 4-6 pulses, yielding the relative signal enhancement of the 20 ns component to about fourfold.

Finally we want to ask the following question: what will be the optimal internal RR in the burst if we want to separate the signal of a probe with fluorescence lifetime of $20 \mathrm{~ns}$ from the background of shorter fluorescence lifetimes. We consider background fluorescence lifetimes of $0.5 \mathrm{~ns}, 1 \mathrm{~ns}$, $2 \mathrm{~ns}$, and $5 \mathrm{~ns}$, respectively. Figure 5 shows the ratio of the initial intensity signal as function of burst internal RR for 2, $3,4,5$ and 6 pulses in the burst. Interestingly the ratios for various fluorescence lifetimes of the background have distinct maxima that depend on the background lifetime and also the number of pulses in the burst. The enhancement clearly can be adjusted for various fluorescence lifetimes of the sample and background (relative enhancement depends on the ratio of fluorescence lifetimes of the sample to the lifetime of the background) and in the presented case is a little over 2 for a $5 \mathrm{~ns}$ background lifetime for 3 and more pulses in the burst. Interestingly the optimal RR in the burst for a $20 \mathrm{~ns}$ sample lifetime and a $5 \mathrm{~ns}$ background is below $200 \mathrm{MHz}$.

Increasing the RR lowers the ratio, and that for infinitely high RR (continuous excitation) should be back to 1. For shorter lifetimes of the background, the enhancement significantly increases (to a value close to fivefold for lifetimes shorter than $1 \mathrm{~ns}$ ) but shifts to higher RR in the burst. For 3-4 pulses and a background lifetime of $1 \mathrm{~ns}$ or less the optimal $\mathrm{RR}$ in the burst are above $300 \mathrm{MHz}$.

\section{Experiment}

The burst of pulsewith a RR of $80 \mathrm{MHz}$ in the burst can be obtained with commercially available electronics as previously described [11]. In figure 6 we are presenting experimental results for intensity decays measured with 10 pulse bursts with an internal RR of $80 \mathrm{MHz}$ for ADOTA ( 20 ns), Rhodamine 6G-R6G (3.4ns), and Erythrosine B-ErB $(0.4 \mathrm{~ns})$ in ethanol. The initial fluorescence signal of ADOTA can be enhanced about twofold as compared to the single pulse while the signals from R6G and ErB are practically unchanged. To improve signal enhancement, it will be necessary to generate pulses with higher RR. Most commercial systems typically allow the lowering of the pulse RR down to $\mathrm{kHz}$ range but the upper limit is typically $80-90 \mathrm{MHz}$. Also it is important to realize that a 20 ns fluorescence lifetime should be measured with a RR of $10 \mathrm{MHz}$ or better lower allowing pulse separation of $100 \mathrm{~ns}$ or more to avoid a problem frequently called cyclic excitation.

For simplicity, consider a source of pulses with a low RR of $1 \mathrm{MHz}$ (1000 ns separation between pulses). It is important to note that increasing pulse power will increase overall signal but will not change the signal-to-background ratio. One way to enhance the signal-to-background ratio would be to transform each pulse to a burst of 2 or more pulses separated by constant delay within the burst. In principle, separating a single pulse into $\mathrm{n}$ pulses will lower the power of each individual pulse in the burst. In the perfect case, the sum of powers from each individual pulse should be equal to the power of initial pulse. Each burst of pulses will be separated by $1000 \mathrm{~ns}$ (dictated by the source RR). The concept is schematically shown in figure 7 . The primary pulse source generates pulses that are separated by time $\mathrm{T}$ corresponding to $1 / \mathrm{RR}$. A box device generates pulse bursts separated by time $\mathrm{T}$ with a set number of pulses in the burst spaced by a time much smaller than T. Such a burst generator can be constructed in various ways. In this report, we are presenting and testing two approaches:

\section{1. Using delay lines}

Figure 8 shows the simple system that allows splitting/dividing the single pulse to multiple pulses of desirable delay. The incoming pulse is partially reflected on mirror $\mathrm{M}_{1}$ and 

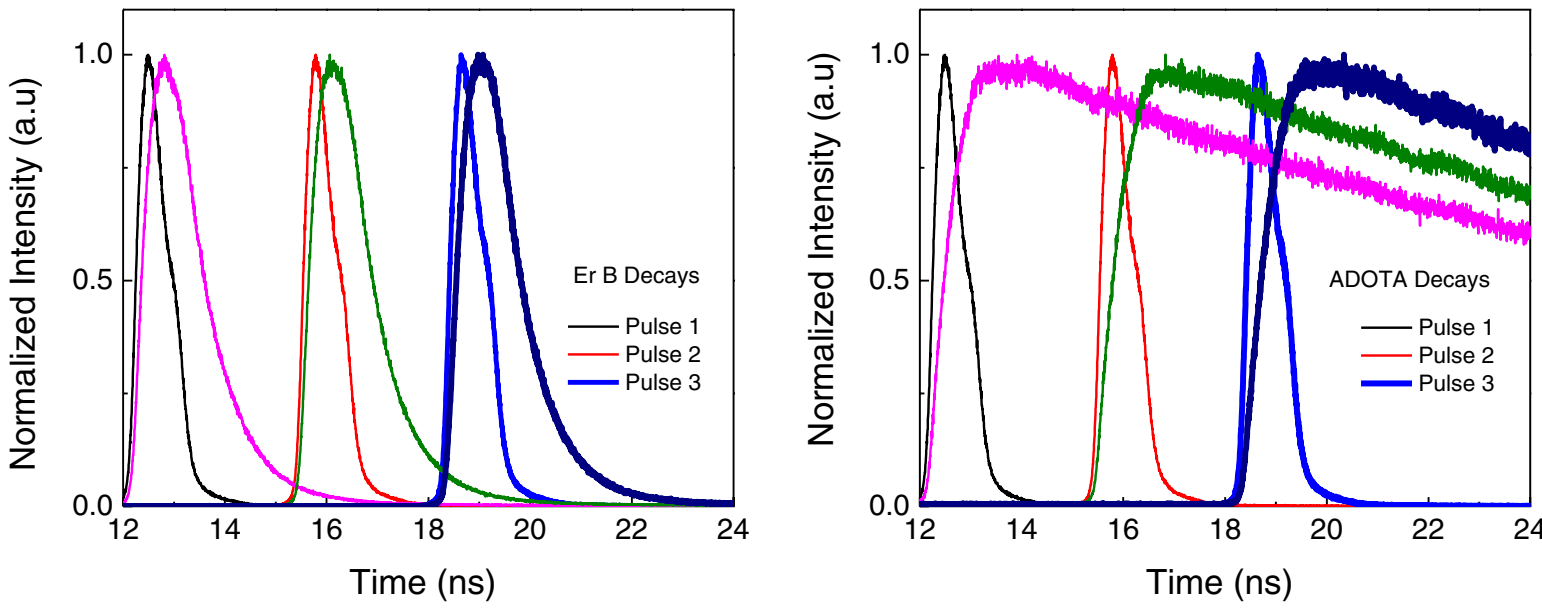

Figure 10. Intensity decays measured with individual pulses for ErB (left) and ADOTA (right).

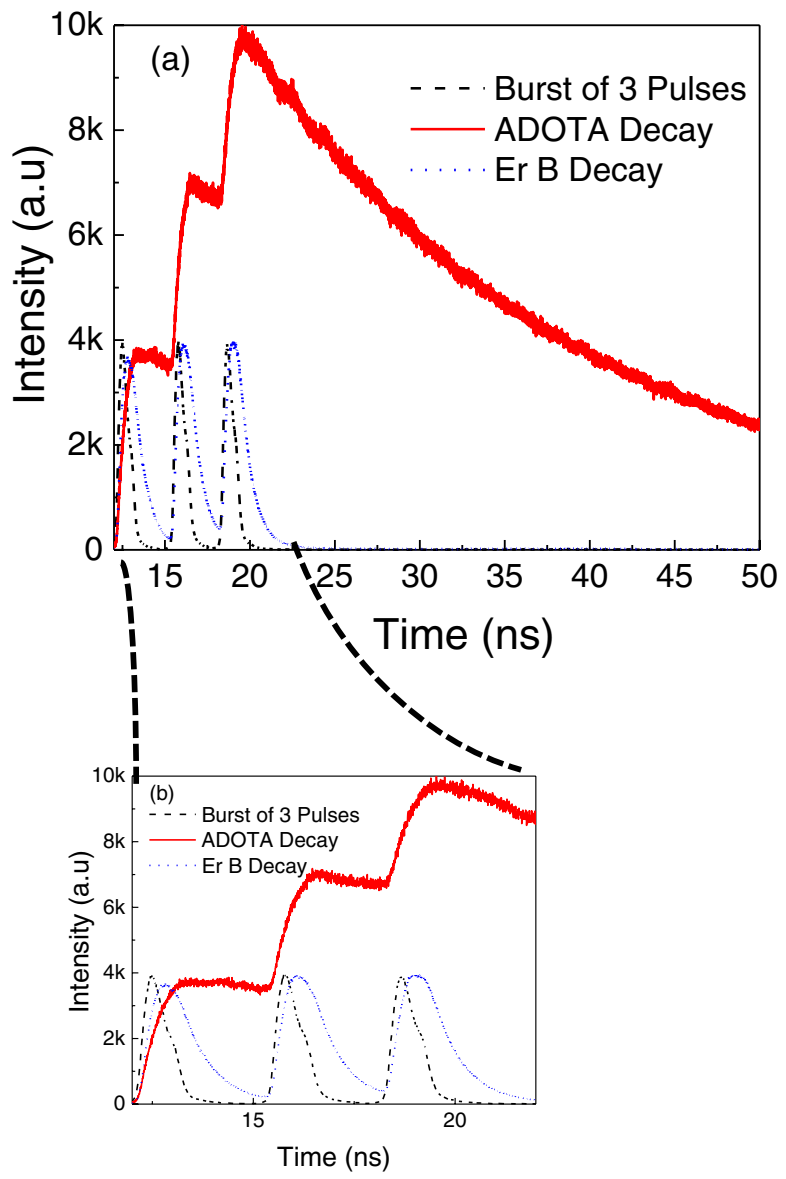

Figure 11. Experimental results with ADOTA (20ns) and ErB $(0.4 \mathrm{~ns})$ with three pulse consecutive excitation.

transmitted by mirror $\mathrm{M}_{1}{ }^{\prime}$. The remaining part of the pulse is then reflected at the mirror $\mathrm{M}_{2}$ and $\mathrm{M}_{2}{ }^{\prime}$ and finally $\mathrm{M}_{1}{ }^{\prime}$ to go on the same line with the first part of the pulse. The operation can be repeated on mirrors $\mathrm{M}_{3} \ldots$ etc. Each consecutive pulse will be delayed proportionally to the distances $a_{\mathrm{i}}$ between the mirrors. If each of the distances $a_{1}, a_{2}, .$. is about $45 \mathrm{~cm}$ then the total pulse delay for the first delay line will be $3 \mathrm{~ns}$. For the next pulse $6 \mathrm{~ns}$, etc. (each consecutive pulse travels each distance $a_{\mathrm{i}}$ twice).
We generated bursts of three pulses using clear glasses (for example microscopy slides) and output from a Picoquant laser diode $485 \mathrm{~nm}$ operating with RR of $1 \mathrm{MHz}$. In such a way, the output pulse power in each line (each pulse in the burst) is lower than $10 \%$ of original power of the pulse. The relative intensity of each pulse can be conveniently regulated by neutral density filters inserted between consecutive mirrors $\mathbf{M}_{n}$ and $\mathbf{M}_{n}^{\prime}$. In figure 9 we present all three pulses generated simultaneously (black dashed line) and each pulse individually by closing two remaining lines noted with points of different color.

We can measure intensity decays with each pulse separately as shown in figure 10 for ErB and ADOTA respectively. The measurement with bursts of three pulses is shown in figure 11 . In this case the initial intensity of ADOTA after three pulses is increased over 2.5-fold while the initial intensity of ErB practically did not change. It is clear that already three pulses gives an excellent enhancement of ADOTA over the shortlived background. The measured fluorescence lifetimes are as expected: $20 \mathrm{~ns}$ for ADOTA and $0.4 \mathrm{~ns}$ for ErB.

\subsection{Using fiber optics of different length}

The concept for using fiber optics is shown in figure 12 . A single pulse is coupled to a bunch of fiber optics. Each fiber optic carries part of the light intensity but has length that differs by $\Delta L$. As pulses exit from each fiber on another end of the bundle they will be delayed proportionally to the length differences. Typically a length of about $0.6 \mathrm{~m}$ results in about $3 \mathrm{~ns}$ delay. In our case we were using five $500 \mu \mathrm{m}$ fibers bundled together. The measured pulses and intensity decays for ErB and ADOTA obtained with five fibers bundled together are presented in figure 13. It is important to stress that for our experiment, we were using five pieces of commercially available fiber optics that were cut to different lengths. In such a crude construction, it was difficult to couple comparable intensities to each fiber. Also, on the output the excitation spots were visually separated. This does not alter results; confirming that pulses do not have to excite exactly the same volume. Excitation at slightly different volumes could be a 


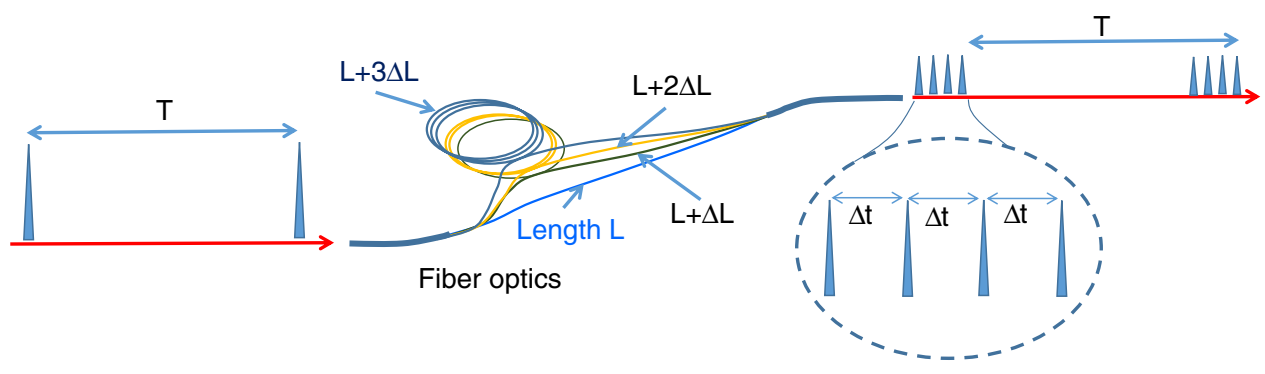

Figure 12. Concept for using fiber optics bundle for generating busts of pulses.

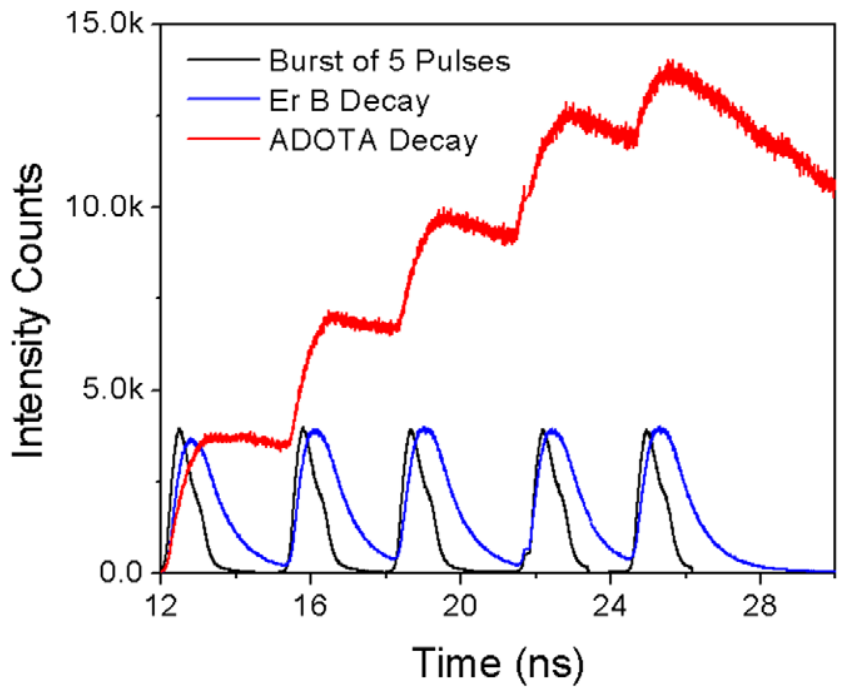

Figure 13. Intensity decays generated with bundle of five fiber optics of different lengths.

disadvantage for microscopy but could be very advantageous for sensing and diagnostics since this may allow for gentle sample excitation and less photobleaching and prevent problems arising from possible saturation. Also for microscopy, the output of fiber bundle can be a single fiber allowing for perfect spot alignment.

Finally using the system in figure 8 with three pulses, we tested fluorophore photostability using a solution of fluorescein using single pulse and three pulses. The energy of a single pulse was adjusted to have input power exactly equal to the cumulative power of three pulses. The results in figure 14 show the photobleaching traces for a single pulse and three pulses. The photostability with three pulses is slightly better probably due to gentler excitation.

\section{Conclusions}

We presented simple ways for generating a burst of pulses of desirable RR in the burst. Such bursts of excitation pulses have been proven to allow for excellent signal enhancement for long-lived probes. The signal enhancement for a longlived dye can be optimized depending on the fluorescence lifetimes of the probe and background by adjusting the internal RR in the burst. Already for a fluorescence lifetime of $20 \mathrm{~ns}$, the signal can be enhanced $500 \%$ over the short lived background ( $<1 \mathrm{~ns})$, and for lifetimes of the probe longer than

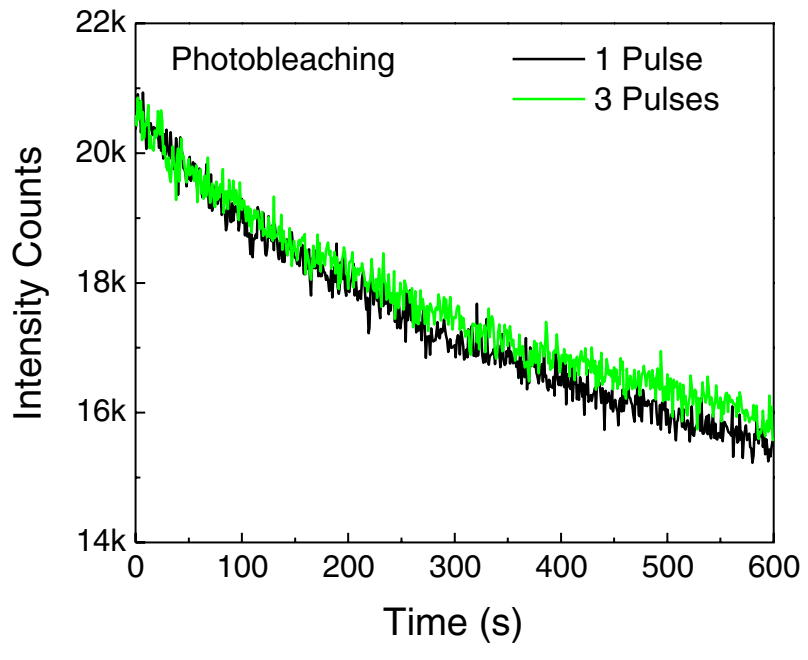

Figure 14. Fluorescein photostability using one pulse (black) and three pulses (green) of equal intensity power.

$20 \mathrm{~ns}$, the enhancement can be much greater. This technology can be very useful in applications to fluorescence based sensing, biomedical diagnostics, and cellular and tissue imaging.

\section{Acknowledgments}

This work was supported by the NIH grant R01EB12003 (ZG) and NSF grant CBET-1264608(IG). We also acknowledge the INFOR support from TCU.

\section{References}

[1] Lakowicz J R 2006 Principles of Fluorescence Spectroscopy 3rd edn (Revised) (New York: Springer Science + Business Media, LLC)

[2] Valeur B and Berberan-Santos M 2012 Molecular Fluorescence: Principles and Applications 2nd edn (Weinhein Wiley-VCH Verlag GmbH \& Co, KGaA)

[3] Jameson D M 2014 Introduction to Fluorescence (Boca Raton, FL: CRC Press, Taylor and Francis Group)

[4] Haugland R B 1996 Handbook of Fluorescent Probes (Molecular Probes, Inc)

[5] Nolting D D, Gore J C and Pham W 2011 Near-infrared dyes: probe development and applications in optical molecular imaging Curr Org Synth. 8 521-34

[6] Fischer G M, Isomaki-Krondahl M, Gottker-Schnetmann I, Daltrozzo E and Zumbusch A 2009 Pyrrolopyrrole cyanine dyes: a new class of near-infrared dyes and fluorophores Chem. Eur. J. 15 4857-64 
[7] Achilefu S 2010 The insatiable quest for near-infrared fluorescent probes for molecular imaging Angew. Chem. Int. Edn Engl. 49 9816-8

[8] Cubeddu R, Ramponi R, Taroni P, Canti G, Ricci L and Supino R 1990 Time-gated fluorescence spectroscopy of porphyrin derivatives incorporated into cells J. Photochem. Photobiol. B 6 39-48

[9] Periasamy A, Siadat-Pajouh M, Wodnicki P, Wang X F and Herman B 1995 Time-gated fluorescence microscopy for clinical imaging USA Microsc. Anal. 3 19-21

[10] Dahan M et al 2001 Time-gated biological imaging by use of colloidal quantum dots Opt. Lett. 26 825-7

[11] Rich R M et al 2013 Multiple-pulse pumping for enhanced fluorescence detection and molecular imaging in tissue Methods. 66 292-8
[12] Sørensen T J, Thyrhaug E, Szabelski M, Gryczynski I, Gryczynski Z and Laursen B W 2013 AzadioxaTriAnulenium: a long fluorescence lifetime fluorophore for large biomolecule binding assay Methods Appl. Fluoresc. 1 025001-7

[13] Rich R M, Mummert M, Gryczynski Z, Borejdo J, Sorensen T J, Laursen B W, Foldes-Papp Z, Gryczynski I and Fudala R 2013 Elimination of autofluorescence in fluorescence correlation spectroscopy using the AzaDiOxaTriAnulenium (ADOTA) fluorophore in combination with time-correlated single-photon counting (TCSPC) Anal. Bioanal. Chem. 405 4887-94

[14] Maliwal B P, Fudala R, Raut S, Kokate R, Sørensen TJ, Laursen BW, Gryczynski Z and Gryczynski I 2013 Long-lived bright red emitting azaoxo-triangulenium fluorophores PLoS One 8 e 63043 